\title{
Genetic Analysis of the Slug Stage of Dictyostelium discoideum
}

\author{
By PETER C. NEWELL* AND FIONA M. ROSS \\ Department of Biochemistry, University of Oxford, South Parks Road, Oxford OXI 3QU, \\ U.K.
}

(Received 18 November 1981; revised 4 January 1982)

\begin{abstract}
During the course of development, cells of Dictyostelium discoideum are able to produce a multicellular body (a 'slug') which is capable of movement over the substratum. This phase, which is induced by production of ammonia by the starving cells, may last for hours or days depending on environmental stimuli. In order to probe the regulatory system controlling formation and duration of the slug phase, mutants were isolated that remained in the slug phase for an extended period. Thirty-two such 'slugger' mutants were analysed by parasexual genetic techniques and placed into 10 complementation groups $(\operatorname{slg} A-s \lg J)$. The linkage groups bearing representatives of these complementation groups were determined by segregation of diploids formed between mutants and tester strains. Phenotypic studies of mutants indicated that members of $\operatorname{sg} D, \operatorname{slg} E$ and $\operatorname{slg} G$ were over-sensitive to the ammonia slug-inducing stimulus.
\end{abstract}

\section{INTRODUCTION}

The normal pathway of development of the cellular slime mould involves the formation of a migratory phase in which the multicellular aggregate is able to move over the substratum with a speed of approximately $1 \mathrm{~mm} \mathrm{~h}{ }^{-1}$. This 'slug' phase lasts for a variable length of time (hours or days) depending on the environmental stimuli (Slifkin \& Bonner, 1952; Newell et al., 1969) and probably has ecological significance in ensuring that fruiting body construction occurs at the surface of the substratum rather than underneath decaying leaves in the natural habitat of forest litter. From studies examining the pattern of formation of several developmentally regulated enzymes (UDPglucose pyrophosphorylase, UDPgalactose epimerase, UDPgalactose transferase and trehalose phosphate synthetase), it appears that while the slug migrates the normal programme of events seen during fruiting body construction is held in abeyance (Newell \& Sussman, 1970; Newell et al., 1972). The stimulus to produce the slug stage is a basic volatile metabolite produced by the aggregating amoebae (Newell et al., 1969) that has been identified by Schindler \& Sussman (1977) as ammonia. Movement of the slug is by production of a slime sheath at the front of the slug to form an extended tube, like a sausage skin, through which the amoebal aggregate moves, leaving a collapsed and empty skin or 'slime trail' behind it (Raper, 1940). The migrating slug responds to light and heat signals of very low intensity, moving towards a light source parallel to the substratum and up a heat gradient of only $0.05{ }^{\circ} \mathrm{C} \mathrm{cm}^{-1}$ (Bonner et al., 1950; Francis, 1964; Poff \& Skokut, 1977). Recent experiments of Fisher et al. (1981) suggest that the operation of these responses requires the production of a factor (called STF) that causes the tip to turn in response to the stimuli. Mutants have been found (Loomis, 1970) which are unable to respond to light signals and which migrate at random in a light gradient. The nature of the light receptor has been extensively studied by Poff and coworkers (Poff et al., 1974; Manabe \& Poff, 1978) but has not yet been rigorously identified. Environmental factors that halt slug migration and restart the development of the fruiting body include a drop in humidity (Slifkin \& Bonner, 1952), the 
presence of phosphate buffer at a pH of 6.0-6.5 and overhead light (Newell et al., 1969). The biochemical mechanism of action of these stimuli is unknown but is thought to involve the production of a factor (appropriately termed 'fruit juice') that has the properties of an aliphatic acid and which may be mimicked by sodium propionate (Sussman et al., 1978).

The formation and termination of the slug phase in Dictyostelium discoideum is an important example of a regulatory system involving the behaviour of a multicellular structure. One approach to understanding this phenomenon is to isolate mutants that are defective in various aspects of the system and to determine the biochemical nature of their lesions. In the study described below, mutants (designated 'sluggers') were isolated which are able to form slugs but are unable to revert to the programme for forming the fruiting body (or revert only after an excessively long period of slug migration). Prior to this study one such mutant (designated KY3) had been reported (Yanagisawa et al., 1967) and this was included in the genetic analysis together with two other slugger mutants (JC2 and JC4) isolated more recently (Sussman et al., 1978).

\section{METHOD S}

Media and chemicals. The composition and methods of preparation of nutrient (SM), $\mathrm{KK}_{2}$ and drug selective media, salts solution (SS) and chemicals were as described previously (Mosses et al., 1975; Ross \& Newell, 1979, 1981; Wallace \& Newell, 1982). The composition of Lower Pad Solution (LPS) is that of Newell et al. (1969).

Growth conditions. All strains of Dictyostelium discoideum were grown in the dark in association with Klebsiella aerogenes at $22^{\circ} \mathrm{C}$, or at the restrictive temperature $\left(27^{\circ} \mathrm{C}\right)$. Bacillus subtilis strain 36.1 , which was used for the selection of $D$. discoideum diploids and for testing for the presence of the bsg markers, has been described previously (Newell et al., 1977a).

Strains. The origins and genotypes of haploid strains are given in Table 1. The strains are all derivatives of NC4. The nomenclature system used is based on that of Demerec et al. (1966). Primary stocks were maintained as spores, either as pure cultures or as synergistic mixtures of mutant and wild-type. Spores were collected in horse serum, dried on silica gel and stored at $4{ }^{\circ} \mathrm{C}$ (Perkins, 1962). Mutants were also stored frozen as amoebae in horse serum containing $5 \%(\mathrm{v} / \mathrm{v})$ dimethyl sulphoxide in plastic straws immersed in liquid nitrogen (Williams \& Newell, 1976). Cloning of stocks by 'streak cloning' and by 'spreader dilution' has been described previously (Wallace \& Newell, 1982).

Formation of diploids and segregation of haploids. Diploid strains were obtained by shaking amoebae of haploid strains bearing non-allelic diploid-selection markers such as $b \operatorname{sg} A 5$ and $t \operatorname{sg} K 21$ and selecting with $B$. subtilis at $27^{\circ} \mathrm{C}$ as described by Newell et al. $(1977 a, b)$. Ploidy was determined on the basis of spore size (diploids being larger) or, where no spores were formed, by cytological staining using the rapid method of Welker $\&$ Williams $(1980 c)$. Diploids were haploidized for segregation analysis using selective drug resistance markers as described by Williams et al. (1974). During the later stages of this study the improved haploidization method of Wallace \& Newell (1982) was used, as this method reduces the tendency of clonal growth effects to bias the segregation pattern. Where suitable drug resistance markers were not available the haploidizing agent benlate was employed (Williams \& Barrand, 1978).

Mutagenesis and isolation of slugger mutants. Freshly harvested amoebae were incubated at $10^{7} \mathrm{ml}^{-1}$ in SS salts solution for $10-45 \mathrm{~min}$ with $N$-methyl- $N^{\prime}$-nitro- $N$-nitrosoguanidine at 200 or $500 \mu \mathrm{g} \mathrm{ml}^{-1}$ so as to give approximately $10 \%$ survival. Mutagenized amoebae were washed three times in SS, plated on $\mathrm{KK}_{2}$ plates without bacteria and allowed to develop. The resulting spores (approximately $5 \times 10^{6}$ per plate) were harvested and stored in $80 \%(\mathrm{v} / \mathrm{v})$ glycerol at $-20{ }^{\circ} \mathrm{C}$. Screening for mutants was carried out by plating spores on SM agar at about 50 spores per plate with an inoculum of $K$. aerogenes and allowing growth of clones to approximately $20 \mathrm{~mm}$ diameter. Clones failing to develop beyond the slug stage were retained, recloned and retested. Because one division, at most, could have occurred during the development of the mutagenized amoebae on the $\mathrm{KK}_{2}$ plates, it is very unlikely that any of the mutants obtained by this technique had a common origin. Because slugger mutants synergize freely, the technique of allowing sporulation before screening for mutants did not prevent isolation of slugger mutants despite their being unable to sporulate in clonal culture. To ensure that classes of slugger mutants that were unable to sporulate by synergism were not missed by the isolation procedure, some experiments employed plating and screening on the SM agar directly after mutagenesis of the amoebae.

Determination of slugger phenotype. For genetic experiments, the slugger phenotype was scored by observing the formation of slugs (rather than mature fruiting bodies) in colonies of 10-20 mm diameter. The colonies were initiated by transfer of amoebae by sterile toothpick on to SM agar inoculated with $K$. aerogenes and grown at $22{ }^{\circ} \mathrm{C}$ in the dark. For comparison of developmental timing, washed amoebae from SM growth plates were 


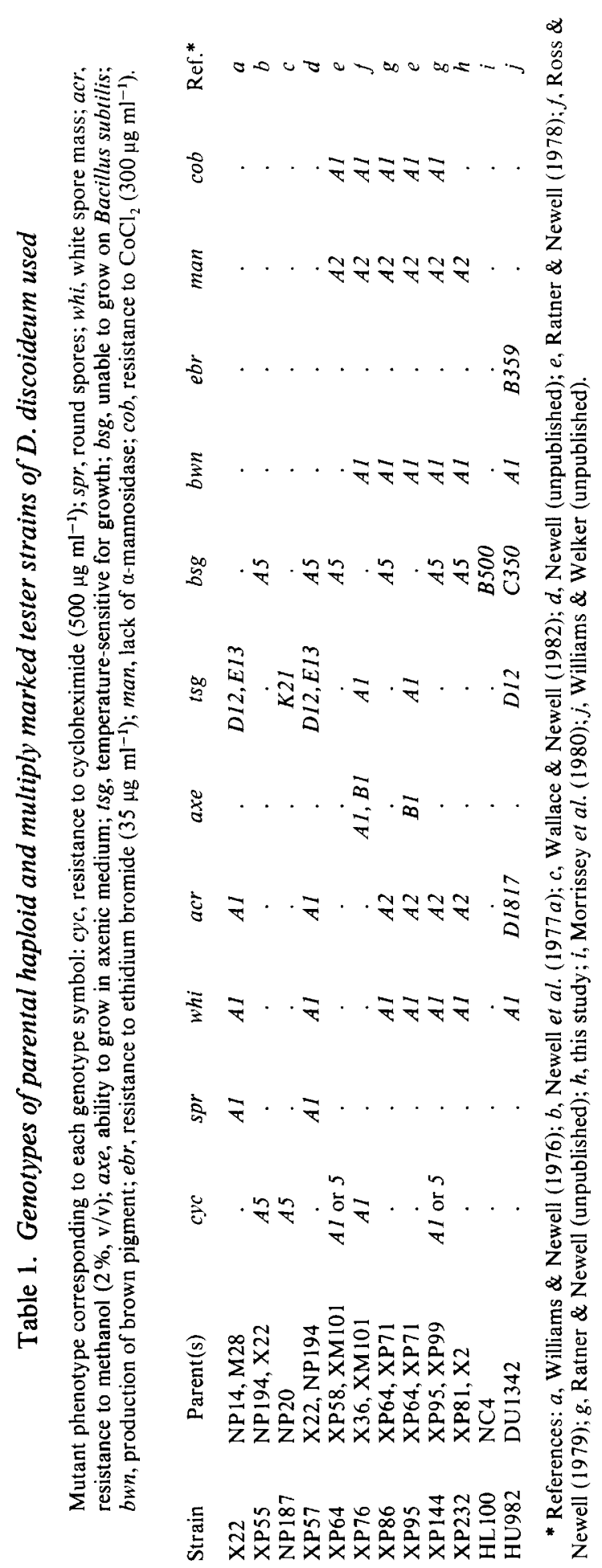


dispensed on to black Whatman no. 29 filters resting on Whatman no. 17 support pads saturated with LPS, as described by Newell et al. (1969). The concentration of phosphate used was $40 \mathrm{~mm}$ and (unless stated otherwise) no upper pads were employed.

\section{RESULTS \\ Isolation of slugger mutants}

The slugger phenotype may be observed in clones growing on lawns of $K$. aerogenes by the failure to complete the normal developmental programme after the slug stage. Using the parental $D$. discoideum strains X22, XP55 and NP187, 32 stable slugger mutants were isolated from eight separate mutagenesis experiments. Additionally, three other mutants (KY3, JC2 and JC4) isolated by M. Sussman in the wild-type (NC4) background were included in this study. All of these mutants were found to synergize with the parental type and stocks were kept of mixed wild-type and mutant spores formed by synergistic development. Most of the mutants formed slugs that were fully capable of migration but a few formed only upright slugs or 'fingers' as though they were blocked at the stage just before that of production of the horizontal slug form. Mutants that remained in the slug phase longer than normal but were then fully capable of forming fruiting bodies were not retained unless the duration of the slug mode was longer than $24 \mathrm{~h}$.

The parental strains used for isolating the slugger mutants possessed either temperaturesensitive growth $(t s g)$ or Bacillus subtilis sensitivity $(b s g)$ mutations so that diploids could be selected when required for the subsequent genetic studies. The mutants KY3, JC2 and JC4, however, lacked any diploid selector. To enable genetic studies on these mutants, tsg mutations were selected after mutagenesis and screening for mutants unable to grow at the non-permissive temperature of $27^{\circ} \mathrm{C}$ but able to grow at $22^{\circ} \mathrm{C}$. Although temperaturesensitive mutants normally fail to grow at the non-permissive temperature, whatever bacterial food source is used, it was noticed that in the case of strain NP389 (the $t s g$ derivative of the slugger strain KY3) growth was fully temperature-sensitive on $K$. aerogenes but only partially so on $B$. subtilis.

\section{Complementation analysis}

After fusion of the mutants in pairs or with the wild-type and testing the resultant diploids for the presence of the slugger phenotype, the mutations were found to be recessive and were placed into 10 complementation groups, $\operatorname{slg} A-s \lg J$ (Table 2).

As five of the complementation groups contained both $b s g$-bearing mutants and/or mutants with non-allelic $t s g$ mutations, the majority of fusions could be carried out within and between groups without difficulty. However, in a number of cases (groups $s \lg F, \operatorname{sig} G, \operatorname{sig} H$, $s \lg I$ and $\operatorname{slg} J$ ), in which all representatives of each group were found in the same genetic background, derivatives of the strains had to be constructed bearing other fusion markers by segregation from appropriate diploids (see Table 2). In the cases of $\operatorname{sg} B$ and $\operatorname{sg} G$ (which are linked to the $b s g A$ marker on linkage group III) the required slugger derivatives lacking the $b s g A$ marker could not be simply constructed by segregation. Instead, they were made by selecting haploids that contained $t s g K 21, b s g A 5, s \lg B 401$ (or $s \lg G 407$ ) from diploids which were heterozygous for these markers, followed by reversion of the $b s g A 5$ mutation as described in the analogous study of streamer mutations by Ross \& Newell (1979). These strains [such as NP414 ( $\lg B)$ and NP418 (slgG)] were required for cross-checking the XP55-derived slugger mutants, all of which bear bsgA5. Analogous strain manipulations for $s \operatorname{si} H$ and $\operatorname{slgJ}$ (which are linked to $t s g K$ on linkage group VII) attempting to eliminate the tsgK 21 marker by reversion were not found to be satisfactory for use with complementation studies. As reported by Wallace \& Newell (1982), isolation of revertants (or suppressors) of the $t s g K 21$ locus using selection for growth at the non-permissive temperature very often introduced additional growth and developmental mutations by an unexplained mechanism. In 
Table 2. Complementation groups determined for the slugger mutants

The last column shows derivative strains in which the diploid selector markers ( $b s g A 5$ or $t s g K 2 I$ ) have been exchanged for other selectors (such as $b s g B 500$ from strain HL 100, or bsgC35O from HU982) and, in certain cases, $b s g A 5$ has been reverted. Strains used most frequently to establish complementation groups are indicated by asterisks.

\begin{tabular}{|c|c|c|c|c|}
\hline $\begin{array}{l}\text { Complementation } \\
\text { group }\end{array}$ & $\begin{array}{l}\text { Mutant } \\
\text { name }\end{array}$ & $\begin{array}{l}\text { Mutation } \\
\text { number }\end{array}$ & $\begin{array}{l}\text { Parental } \\
\text { strain }\end{array}$ & $\begin{array}{l}\text { Representative derivatives } \\
\text { with diploid selector } \\
\text { in parentheses }\end{array}$ \\
\hline $\operatorname{slg} A$ & $\begin{array}{r}\text { *NP208 } \\
\text { NP419 } \\
\text { NP437 } \\
\text { NP443 } \\
\text { NP444 } \\
\text { NP446 } \\
\text { NP451 }\end{array}$ & $\begin{array}{l}\text { slgA400 } \\
\text { slgA409 } \\
\text { slgA424 } \\
\text { slgA430 } \\
\text { slgA431 } \\
\text { slgA433 } \\
\text { slgA438 }\end{array}$ & $\begin{array}{l}\text { NP187 } \\
\text { XP55 } \\
\text { NP187 } \\
\text { NP187 } \\
\text { NP187 } \\
\text { NP187 } \\
\text { NP187 }\end{array}$ & ${ }^{*} \mathrm{XP} 189\left(t s g D 12 t s g E 13 t s g K^{+}\right)$ \\
\hline $\operatorname{slg} B$ & $\begin{array}{r}\text { *NP347 } \\
\text { NP388 } \\
\text { NP407 } \\
\text { NP445 } \\
\text { NP454 }\end{array}$ & $\begin{array}{l}s \lg B 401 \\
s \lg B 403 \\
s \lg B 405 \\
s \lg B 432 \\
\operatorname{sig} B 441\end{array}$ & $\begin{array}{l}\text { XP55 } \\
\text { XP55 } \\
\text { XP55 } \\
\text { NP187 } \\
\text { NP187 }\end{array}$ & $\mathrm{XP} 243\left(b s g B 500 t s g K^{+}\right)$ \\
\hline $\operatorname{slg} C$ & $\begin{array}{l}\text { *NP351 } \\
\text { JC2 } \\
\text { JC4 } \\
\text { NP426 } \\
\text { NP436 } \\
\text { NP438 }\end{array}$ & $\begin{array}{l}\text { slgC402 } \\
\text { slgC } 701 \\
\operatorname{sig} C 702 \\
\operatorname{slg} C 413 \\
\operatorname{sig} C 423 \\
\operatorname{slg} C 425\end{array}$ & $\begin{array}{l}\text { X22 } \\
\text { NC4 } \\
\text { NC4 } \\
\text { NP187 } \\
\text { NP187 } \\
\text { NP187 }\end{array}$ & $\begin{array}{l}\text { NP396 (tsg-404) } \\
\text { NP417 (tsg-406) }\end{array}$ \\
\hline $\operatorname{slg} D$ & KY3 & $\operatorname{slg} D 700$ & $\mathrm{NC} 4$ & *NP389 (tsg-400) \\
\hline $\operatorname{slg} E$ & $\begin{array}{r}\text { NP392 } \\
\text { NP422 } \\
\text { NP429 }\end{array}$ & $\begin{array}{l}s \lg E 404 \\
\operatorname{slg} E 410 \\
\operatorname{slg} E 416\end{array}$ & $\begin{array}{l}\text { XP55 } \\
\text { NP187 } \\
\text { NP187 }\end{array}$ & $\begin{array}{l}\mathrm{XP} 183\left(t s g A 1 b s g A^{+}\right) \\
\mathrm{XP} 267\left(b s g B 500 t s g K^{+}\right)\end{array}$ \\
\hline $\operatorname{slg} F$ & $\begin{array}{l}\text { NP428 } \\
\text { NP430 } \\
\text { NP440 } \\
\text { NP441 } \\
\text { NP448 } \\
\text { NP452 } \\
\text { NP453 }\end{array}$ & $\begin{array}{l}\text { slgF415 } \\
\text { slgF417 } \\
\text { slgF427 } \\
\text { slgF428 } \\
\text { slgF435 } \\
\text { slgF439 } \\
\text { slgF440 }\end{array}$ & $\begin{array}{l}\text { NP187 } \\
\text { NP187 } \\
\text { NP187 } \\
\text { NP187 } \\
\text { NP187 } \\
\text { NP187 } \\
\text { NP187 }\end{array}$ & $\begin{array}{r}{ }^{*} \mathrm{XP} 228\left(b s g B 500 t s g K^{+}\right) \\
\left.\text {XP243 (bsgB500 tsgK }{ }^{+}\right)\end{array}$ \\
\hline $\operatorname{slg} G$ & $\begin{array}{r}\text { NP409 } \\
\text { *NP410 }\end{array}$ & $\begin{array}{l}\text { slgG407 } \\
\text { slgG408 }\end{array}$ & $\begin{array}{l}\text { XP55 } \\
\text { XP55 }\end{array}$ & NP418 (tsgK2l bsgA $\mid$ reverted $\mid)$ \\
\hline $\operatorname{slg} H$ & *NP449 & $\operatorname{slg} H 436$ & NP187 & XP317 (bsgC350 tsgK21) \\
\hline $\operatorname{slg} I$ & *NP435 & $\operatorname{slg} 1422$ & NP187 & XP241 (bsgA5 tsgK $\left.{ }^{\dagger}\right)$ \\
\hline $\operatorname{slg} J$ & *NP439 & $\operatorname{slgJ426}$ & NP187 & $\mathrm{XP} 258(b s g A 5 t s g K 2 l)$ \\
\hline
\end{tabular}

some instances the slg mutation was reverted (or suppressed) along with reversion (or suppression) of the $t s g K 21$ marker, and in one case the $s / g$ mutation was rendered temperature-sensitive (allowing sporulation at $22{ }^{\circ} \mathrm{C}$ but halting development at the slug stage above $26.5^{\circ} \mathrm{C}$ ). Consequently, use was made of the recently discovered $b s g C$ mutation on linkage group IV (Welker \& Williams, 1980 $a$ ) to allow diploids to be selected between $b s g C$ derivatives of $\operatorname{slg} H$ and $b s g A$ derivatives of $\operatorname{slg} J$ at $22^{\circ} \mathrm{C}$.

\section{Linkage analysis of slugger loci}

Tester strains. Because the slugger mutations were introduced into several different parental strains, variously marked tester strains were required for linkage analysis. As this study was conducted over a period of several years, some of the early experiments with mutants of groups $\operatorname{slg} A, \operatorname{sg} B$ and $\operatorname{sig} C$ used tester strains such as XP57, XP64 and XP86, which were later superseded. In these cases, linkage analysis was repeated with the more 


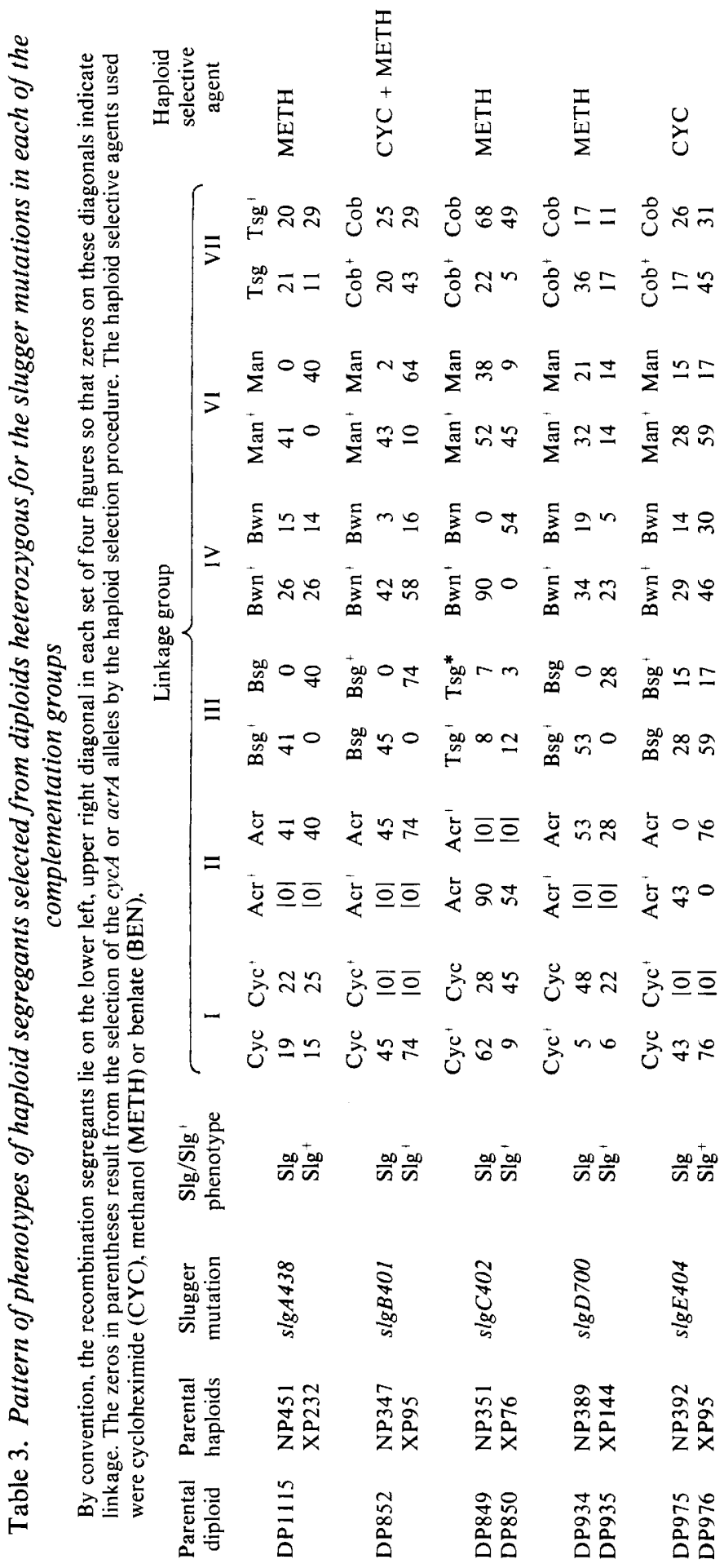




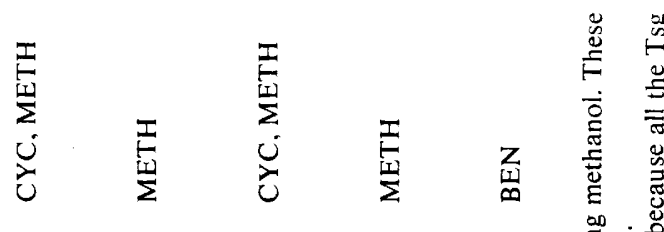

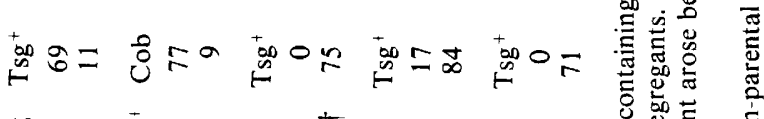

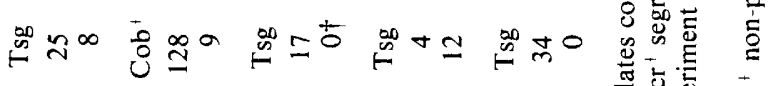

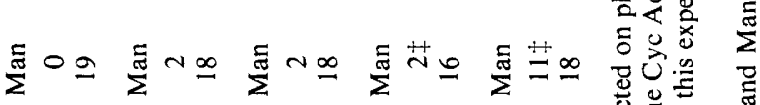
市

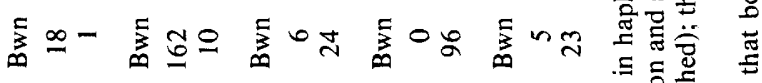

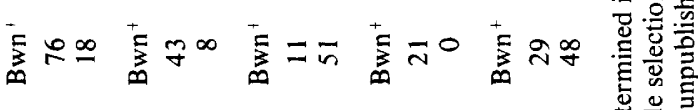
禹○

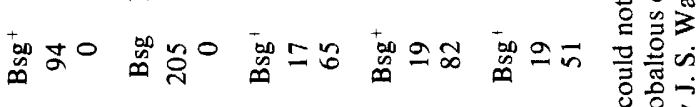

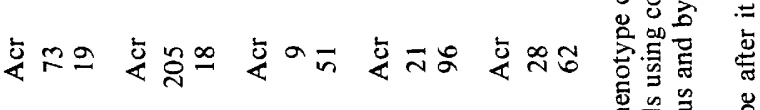

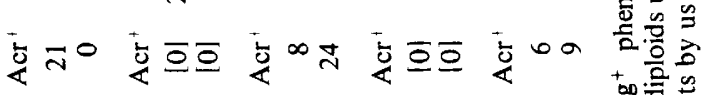

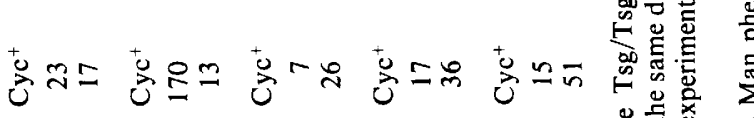
湈

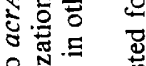

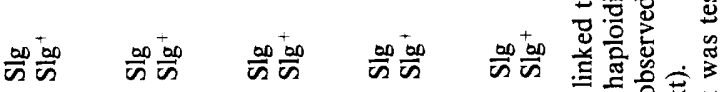

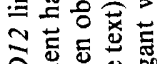

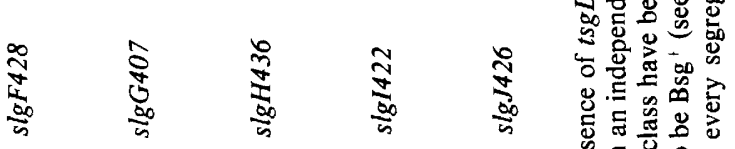

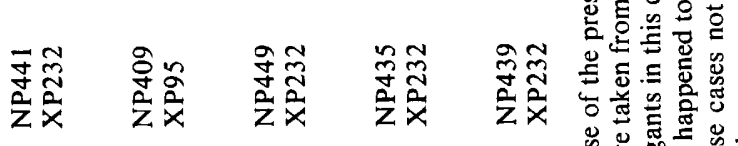

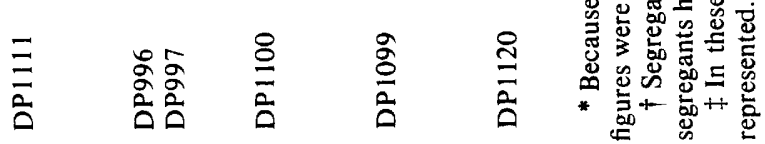


Table 4. Assignment of complementation groups slgA-slgJ to linkage groups, based on the pattern of segregation shown in Table 3

\begin{abstract}
The distinction between linkage groups III and VI in the assignment of $\operatorname{sg} A, \operatorname{sig} F$ and $\operatorname{sig} G$ is ambiguous (possibly due to translocation). The SlgH phenotype requires both linkage groups III and VII from the mutant strain as described in the text.
\end{abstract}

$\begin{array}{cl}\text { Complementation group } & \text { Linkage group } \\ & \text { III/VI } \\ \operatorname{sig} A & \text { III } \\ \operatorname{sig} B & \text { IV } \\ \operatorname{sig} D & \text { III } \\ \operatorname{sig} E & \text { II } \\ \operatorname{sig} F & \text { III/VI } \\ \operatorname{sig} G & \text { III/VI } \\ \operatorname{sig} H & \text { III }+ \text { VII } \\ \operatorname{sigI} & \text { IV } \\ \operatorname{sig} J & \text { VII }\end{array}$

recently constructed tester strains that allowed simpler interpretation of the phenotypic pattern; for brevity, only the latter results are shown in Table 3. Derivatives of parental strain XP55 were fused with tester strain XP95 which has been described previously (Ratner \& Newell, 1978). Tsg derivatives of the slugger mutants obtained from M. Sussman were fused with either XP76 or XP144. Derivatives of parental strain NP187 were fused with XP232 which was specifically constructed as the appropriate tester for this strain.

Segregation of diploids. In most cases, haploidization of the diploids constructed between the slugger mutants and the tester strains was achieved by selection for the acrA marker using agar plates containing $2 \%(\mathrm{v} / \mathrm{v})$ methanol. In cases where such selection caused problems, such as for $\operatorname{slg} E$ which is linked to $a c r A^{+}$on linkage group II, the $c y c A$ selector was used with plates containing $500 \mu \mathrm{g}$ cycloheximide $\mathrm{ml}^{-1}$. In the later phase of the study the haploidizing agent benlate was also employed at $20 \mu \mathrm{g} \mathrm{ml}^{-1}$ (Williams \& Barrand, 1978). Although this agent generally gave good segregation patterns for most linkage groups, it showed a considerable bias for selection of the acrA marker on linkage group II due to cross-resistance of this marker for benlate resistance (see results for $\operatorname{slgJ}$, Table 3 ).

Complexities of the phenotypic pattern. For most of the data in Table 3, the pattern of phenotypes allows unambiguous assignment of the linkage groups of the slugger mutants (Table 4). However, in certain cases the pattern is reproducibly complex. For example:

(a) Due to co-segregation of linkage groups III and VI in experiments employing $\operatorname{slg} A, \operatorname{sig} F$ and $\operatorname{slg} G$ mutants, the phenotypic pattern of segregants cannot distinguish in which of these two linkage groups the mutations are located. Such co-segregation has been noticed in previous studies with related strains (Ross \& Newell, 1979; Welker \& Williams, 1980 b) and may be due to a translocation in the strains used. The effect is seen most strongly with the parental strain NP187 but usually to a lesser degree with strain XP55, as may be noticed with some of the XP55-derived slugger mutants in Table 3. In the case of $s / g F$, all seven mutants isolated were in the NP187 background and showed absolute co-segregation of linkage groups III and VI. In the case of $\operatorname{slg} A$, six of the seven mutants isolated were also in this strain and produced similar results. The seventh $\operatorname{sig} A$-bearing strain was isolated in an XP55 background. Linkage analysis of this strain again showed nearly complete co-segregation of linkage groups III and VI. In 153 segregants tested with this strain only one exception was found to the III/VI co-segregation pattern and (after rigorous examination of its phenotype) this indicated that $\operatorname{sig} A$ is linked to group VI rather than group III. In the case of $\operatorname{sig} G$, the data from two out of 223 segregants indicated that this mutation is located on linkage group III rather than VI. However, in view of the relatively small numbers of segregants involved these assignments must be stated tentatively. 
(b) Strong bias may at times be noticed in the phenotypic pattern. However, as long as one or two segregants of each of the four phenotypic classes can be rigorously established for any particular linkage group, then the bias may not influence the interpretation of the linkage assignment. In some cases, however, the complete absence of a class of segregant could be interpreted as evidence for the involvement of more than one linkage group for the slugger phenotype, or the presence of slugger suppressor mutations in the tester strain being used. An example of this type of effect was obtained with haploidization of diploid DP1105, constructed from the slugger strain NP433 (derived from NP187) and tester strain XP232. The pattern of phenotypes produced by this diploid strain with cycloheximide or methanol selection showed no clear linkage to any particular linkage group but three classes of segregant on linkage group IV. The failure to find only two classes of segregant for any of the marked linkage groups suggests that the $s l g$ mutation being studied is on linkage group $\mathrm{V}$ (which is as yet unmarked). However, the appearance of only three classes of segregant on linkage group IV (with an absence of the slugger non-parental class) also suggests that the parental linkage group IV from the slugger strain may be essential for the slugger phenotype. This makes the analysis of this particular pattern unresolvable with our present lack of understanding of linkage group $\mathrm{V}$, and this mutant has not been included in the data presented in Tables 2 and 3 of this study. A basically similar, but more analysable result, was found with $\operatorname{slgH}$. In this case, three classes of phenotypes were found for linkage groups III and VII, the missing classes being Slg Bsg on III and Slg Tsg ${ }^{+}$on VII (see Table 3). This finding suggests that both linkage groups III and VII of the slugger strain are required for expression of the slugger phenotype, possession of only one of these linkage groups giving a phenotype that is wild-type. In principle, such complex linkage results may cause

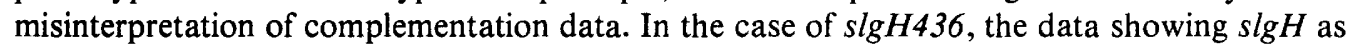
a separate complementation group has been retained, as the only other slugger showing any requirement for linkage group VII $(s l g J)$ did not show such effects and was clearly phenotypically distinct from the $s \lg H$ mutation. The status of $s \lg H$ remains tentative, however, as the possibility cannot presently be ruled out that a mutation on linkage group III or VII which gives only a partial effect in $\operatorname{slg} H$ is able to produce a complete slugger phenotype in other strains.

Sometimes an apparent bias and missing phenotypic classes may be seen only with one particular selector. In the case of $s l g F$ there was a strong bias for the Slg class of segregants when using selection of haploids with cycloheximide, which in some segregations led to missing classes of segregant. Fortunately, with selection on methanol plates this bias was not apparent, and both Slg Cyc and Slg $\mathrm{Cyc}^{+}$classes freely segregated. The data shown in Table 3 for $\operatorname{slg} F$ are the combined data from an experiment that employed both methanol and cycloheximide selection plates (independently) so that the bias due to the cycloheximide (which was absolute in this experiment) appears as a single zero in the $\mathrm{Slg}^{+} \mathrm{Acr}^{+}$class. Because of the results found with methanol, any explanation of the cycloheximide bias in terms of the need for linkage group I (bearing $c y c A 5$ ) of the parental strain would seem to be ruled out.

\section{Phenotypes of complementation groups $A$ to $J$}

Examination of the phenotypes of the mutants developing on filters in the dark revealed significant and consistent differences between the groups. The phenotypes are shown diagrammatically in Fig. 1. The normal wild-type pattern of development passes through the finger and slug stage for a period that lasts approximately $5 \mathrm{~h}$ under the standard conditions used. In the mutants this period is extended for many hours, in some cases indefinitely. With $\operatorname{sig} A$ mutants a normal migrating slug stage is formed which migrates for about $60 \mathrm{~h}$ before taking on a withered appearance as the slug ages and the cells eventually die. With $\operatorname{sig} B$ the mutants again migrate for long periods and characteristically leave behind a slime trail that appears deformed with a somewhat crystalline appearance. Mutants of $s l g C$ form very large 

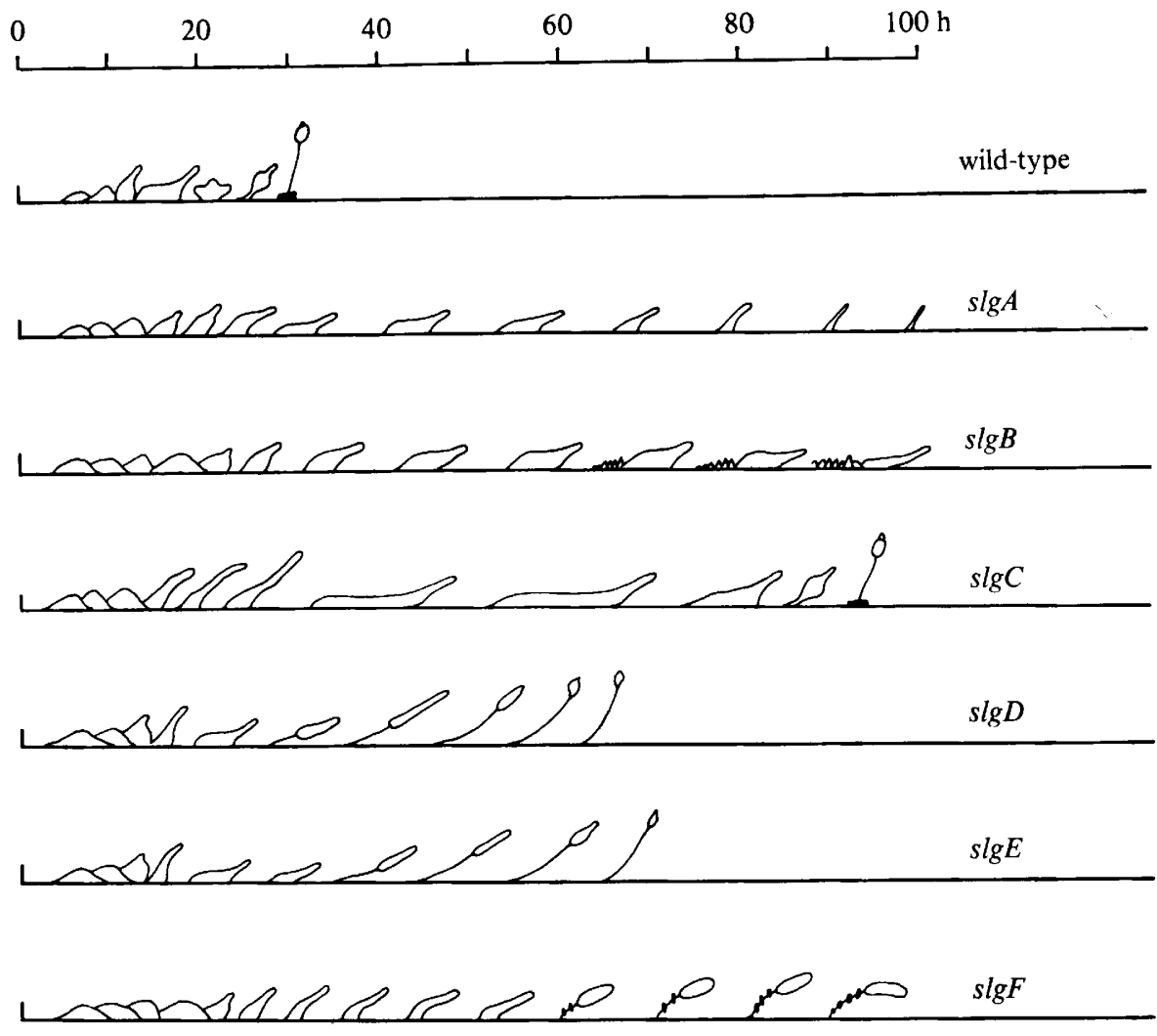

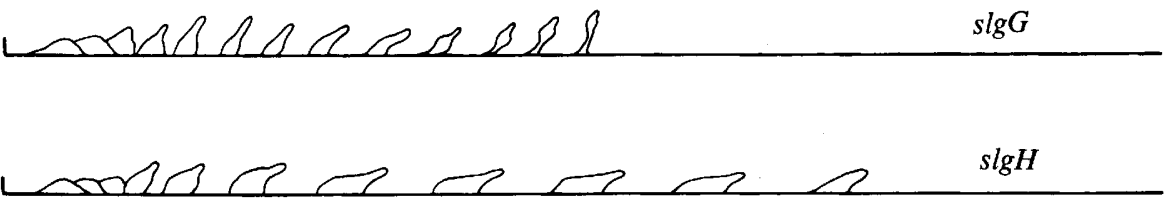
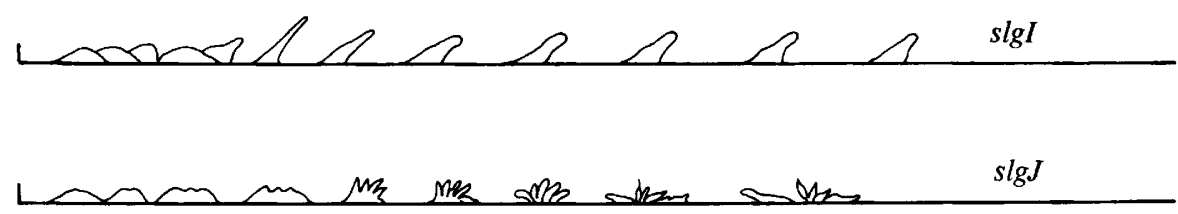

Fig. 1. Schematic representation of the morphology of the developmental stages of the wild-type strain and mutants of complementation groups $\operatorname{sig} A-\operatorname{sig} J$. For all except $\operatorname{slg} F$ the timing is that seen at $22^{\circ} \mathrm{C}$ in the dark, the cells being incubated at a density of $5 \times 10^{6} \mathrm{~cm}^{-2}$ on $47 \mathrm{~mm}$ diameter Whatman no. 29 filter discs (cut into quarters) supported by Whatman no. 17 pads saturated with LPS containing 40 mM-phosphate buffer as described by Newell et al. (1969). Under these conditions slgF cells aggregate poorly and so the timing shown is that seen in clones growing on SM agar inoculated with $K$. aerogenes. Note that for most groups the morphology and duration of the stages is influenced to some extent by the cell population density. This effect is particularly noticeable for $\operatorname{slg} D$ and $s \lg E$ which, under conditions of higher density on SM agar, can form greatly extended aerial slime trails. The bead-like structures formed on the trails of slugs of $s \lg F$ mutants are drops of liquid that do not contain spores.

The strains used as representatives of the different $s \lg$ complementation groups were: $\operatorname{slg} A$, NP208; $s \lg B$, NP347 and NP388; $s \lg C$, NP351 and NP396; $\operatorname{sg} D$, NP389; $\operatorname{slgE,~NP392;~} \lg F$, NP452 and NP453; slgG, NP409 and NP410; slgH, NP449; slgI, NP435; slgJ, NP439. 
slugs that migrate normally for up to $60 \mathrm{~h}$. Two representatives of this complementation group (JC2 and JC4) have been examined in detail by Sussman et al. (1978) and found to synthesize the developmental enzymes (UDPglucose pyrophosphorylase, UDPgalactose epimerase and UDPgalactose transferase) that would be expected for the wild-type strain induced to form an extended slug migration phase. We confirmed the finding of Sussman et al. (1978) that the defect in $\operatorname{slg} C$ mutants was not principally an over-sensitivity to the slug migration stimulus (ammonia) but probably the failure to produce normal amounts of the fruit-inducing stimulus (fruit juice), since these mutants were induced to form fruiting bodies by addition of sodium propionate to the medium, as described by Sussman et al. (1978). The phenotype of $\operatorname{slg} D$ [only one member of which (KY3) has been observed so far] has been detailed previously by Yanagisawa et al. (1967). The phenotype of KY3 is highly dependent on the cell density: at low cell density $\left(5 \times 10^{5} \mathrm{~cm}^{-2}\right)$ it forms fruiting bodies with almost normal appearance in the normal time (although with only prespores being formed in the spore masses), but at high cell density $\left(1 \times 10^{7} \mathrm{~cm}^{-2}\right)$ it forms slugs which migrate for an extended period, eventually producing aerial 'slugs' supported by stalk-like slime trails. By switching staled substrata from the wild-type and the mutant, it has been established that KY3 is over-sensitive to the slug-forming stimulus (ammonia) (Newell \& Sussman, cited in Newell, 1971; Schindler \& Sussman, 1979) and this would explain the density dependence of the phenotype first noted by Yanagisawa et al. (1967). Mutants of $\operatorname{sg} E$ are phenotypically very similar to mutant $\mathrm{KY} 3$ and show a similar density dependence and formation of aerial slugs. Using the technique of ammonia removal by the enzyme $\mathrm{NAD}^{+}$-linked L-glutamate dehydrogenase with NADH and 2-oxoglutarate in the substratum or sprayed on to the slugs, as described by Schindler \& Sussman (1977), slgE mutant NP392 was also found to resemble KY3 in forming fruits under conditions in which ammonia was not allowed to accumulate. Genetically, however, these two mutants are clearly distinct, KY3 $(s \lg D)$ being on linkage group III and complementing NP392 ( $\lg E$ ) on linkage group II. Because of problems with poor aggregation of mutants of $s / g F$ on filters, the phenotype shown in Fig. 1 is that seen on SM agar. The $s l g F$ mutants form trails that lift the slug off the substratum and which with age can collect droplets of fluid along their length. Members of $\operatorname{slg} G$ differ from the other sluggers in only reaching the upright slug (or 'finger') stage and can rarely be induced to migrate. They are also temperature-sensitive such that the fingers tend to collapse into mounds when the temperature is raised above $23-24^{\circ} \mathrm{C}$. Although on SM agar $s \lg G$ mutants do not progress beyond the finger stage, on filters the phenotype is clearly population-density dependent and at decreasing densities an increasing proportion of fingers are able to go on to form fruiting bodies. This formation is accelerated by the ammonia-removal enzyme system and by suspending upper pads saturated with $0.5 \mathrm{M}-\mathrm{KH}_{2} \mathrm{PO}_{4} / \mathrm{K}_{2} \mathrm{HPO}_{4}$ buffer $(\mathrm{pH} \mathrm{6.0)}$ ) above the developing fingers. The $s l g H$ phenotype is distinctive in forming a fat migrating slug that shows no tendency to form fruiting bodies at any population density, while $s l g I$ tends to form blunt-nosed slugs that often migrate in a leaning position rather than horizontal to the substratum. The $s l g J$ phenotype is distinctive in forming a rosette of tiny slugs that branch out from each original aggregate. These are capable of migrating but generally do not move very far on SM agar plates.

Comparative experiments with the ammonia-removal enzyme system indicated that only $\operatorname{slg} D, \operatorname{slg} E$ and $\operatorname{slg} G$ are affected by removal of ammonia and hence over-sensitive to this metabolite, while addition of sodium propionate (the agent that mimics fruit juice) to all groups indicated that only $\operatorname{slg} C$ is induced to fruit by the presence of this chemical.

A general property of the slugger mutants observed is that although the genetic background can strongly influence the phenotypic appearance, the phenotypes of mutants in the same complementation group isolated in the lightly marked parental strains used in this study are very similar. The mutants may often show differences in the size of the slugs formed (this being influenced by factors affecting the territory size) but the characteristics of the particular complementation group are generally still apparent. 


\section{DISCUSSION}

Complementation tests with 34 slugger mutants established 10 complementation groups. As four of these groups contain only one member it would seem likely that not all slugger genes are represented in this study. Assuming that all loci involved are equally mutable, statistical calculations from the complementation data indicate that the probability of there being more than 10 loci is significant $(P=0.38)$ but the probability of there being more than 12 is relatively small $(P=0.03)$.

The pattern of loci found from linkage analysis shows that the slg loci are scattered over four of the seven linkage groups without obvious evidence for gene clustering (although more groups may be associated with linkage group III than with any of the other linkage groups). Such scattering of genes associated with a particular phenotype is probably the normal pattern for this organism as a similar lack of clustering has now been reported for 10 'aggregateless' (agg) loci (Williams \& Newell, 1976; Coukell, 1975, 1977), 10 radiation sensitivity (rad) loci (Welker \& Deering, 1976, 1978; Coukell \& Cameron, 1979) and six streamer (stm) loci (Ross \& Newell, 1979, 1981).

During the linkage analysis, complexities were found in the pattern of segregation of some of the markers. Some of these have been discussed previously (Newell, 1978; Ross \& Newell, 1979; Welker \& Williams, 1980 b). However, one such problem, concerned with the requirement for more than one linkage group for the phenotypic pattern, was not previously encountered in so clear a manner. The finding that mutant NP449 $(s \lg H)$ required both linkage groups VII and III of the slugger strain in order to show the slugger phenotype, may be an example of an interaction of structural or (more probably) regulatory genes, both of which must be mutated for production of the observed aberration. It seems probable that this type of effect is more common than generally stated as many developmental mutations are 'affected' to some extent by the genetic background into which they are placed. Whether the effect of one of the linkage groups that seem to be required is strictly due to a mutation within that linkage group (an 'enhancer gene', for example) or is due to a suppressor gene located in the same linkage group in the tester strain that was used to construct the diploids is not always easily resolvable and may be a question of semantics if the suppressor is found to be widely distributed.

The main purpose of this study was to identify the genetic loci involved with the slugger phenotype, and the nature of the aberrations associated with the mutations was only briefly considered. Because the formation of the slug stage is a behavioural response to a crowded environment that is monitored by ammonia production (formed by breakdown of cellular protein for energy under starvation conditions), any defect leading to over-sensitivity in the monitoring system would lead to excessive stimuli for slug formation. Three of the slugger groups $(\operatorname{sg} D, \operatorname{sg} E$ and $\operatorname{slg} G$ ) show such over-sensitivity and can be induced to fruit by efficient removal of ammonia. The effect of sodium propionate, which was found by Sussman et al. (1978) to stimulate fruiting body formation in the mutants JC2 and JC4, was confirmed and another mutant (NP351) of the same complementation group ( $\operatorname{slg} C$ ) was tested and found to behave in a similar way. Presumably the mutants of this complementation group have a block in the formation of the natural fruit-inducing compound. Another metabolic defect that would be expected to lead to the slugger phenotype would be the over-production of ammonia, giving a dominant slugger phenotype in synergistic mixtures with the wild-type, but so far this class of mutant has not been identified.

The slugger mutants present the opportunity to explore the regulation of a behavioural response at a very simple level. Some, at least, of the defects should be readily resolvable while others may be far more deeply involved with the regulation of development than current thinking will elucidate. In this connection it is interesting that a study of these mutants by Harry MacWilliams of University of Massachusetts, Worcester, U.S.A. (personal communication) has revealed that while most of the mutants show normal ratios of prespore to 
prestalk regions in the slugs (as demonstrated by vital staining with neutral red) the three members of the $\operatorname{sg} E$ group all show an abnormal pattern with the anterior (prestalk) region being very small compared with the other mutants or the wild-type.

We gratefully acknowledge the able technical assistance of Stephanie Rogers, Steven McClue and Jane Hardwicke. We thank Frank Caddick for drawing the figures, Steven Cobbold for early work on some of the mutant phenotypes, Maurice Sussman for the gift of strains JC2 and JC4. Alan Grafen and Nigel Wilkes for making the statistical calculations and Jeannie Wallace for her helpful comments on the manuscript. This work was funded by a grant from the Science Research Council.

\section{REFERENCES}

Bonner, J. T., Clarke, W. W., NeEly, C. L. \& SlifKIN, M. K. (1950). The orientation to light and the extremely sensitive orientation to temperature gradients in the slime mold Dictyostelium discoideum. Journal of Cellular and Comparative Physiology 36, 149-158.

Coukell, M. B. (1975). Parasexual genetic analysis of aggregation deficient mutants of Dictyostelium discoideum. Molecular and General Genetics 142, 119-135.

Coukell, M. B. (1977). Evidence against mutational 'hot-spots' at aggregation loci in Dictyostelium discoideum. Molecular and General Genetics 151 , 269-273.

Coukell, M. B. \& Cameron, A. M. (1979). radE, a new radiation-sensitive locus in Dictyostelium discoideum. Journal of General Microbiology 114, 247-256.

Demerec, M., Adelberg, E. A., Clark, A. J. \& Hartman, P. E. (1966). A proposal for a uniform nomenclature in bacterial genetics. Genetics 54. 61-76.

Fisher, P. R., Smith, E. \& Williams, K. L. (1981). An extracellular chemical signal controlling phototactic behavior by Dictyostelium discoideum slugs. Cell 23, 799-807.

Francis, D. W. (1964). Some studies on phototaxis of Dictyostelium. Journal of Cellular and Comparative Physiology 64, 131-138.

Loomis, W. F. (1970). Mutants in phototaxis of Dictyostelium discoideum. Nature, London 227 , 745-746.

Manabe, K. \& Poff, K. L. (1978). Purification and characterization of photoreducible B-type cytochrome from Dictyostelium discoideum. Plant Physiology 61, 961-966.

Morrissey, J. H., Wheeler, S. \& LOOMIS, W. F. (1980). New loci in Dictvostelium discoideum determining pigment formation and growth on Bacillus subtilis. Genetics 96, 115-123.

Mosses, D., Williams, K. L. \& Newell, P. C. (1975). The use of mitotic crossing-over for genetic analysis in Dictvostelium discoideum: mapping of linkage group II. Journal of General Microbiology 90, 247-259.

NEwELL, P. C. (1971). The development of the cellular slime mould Dictyostelium discoideum: a model system for the study of cellular differentiation. Essays in Biochemistry 7, 87-126.

Newell, P. C. (1978). Genetics of the cellular slime molds. Annual Review of Genetics 12, 69-93.
Newell, P. C. \& Sussman, M. (1970). Regulation of enzyme synthesis by slime mold cell assemblies embarked upon alternative developmental programs. Journal of Molecular Biology 49, 627-637.

Newell, P. C., Telser, A. \& Sussman, M. (1969). Alternative developmental pathways determined by environmental conditions in the cellular slime mold. Journal of Bacteriology 100, 763-768.

Newell, P. C., Franke, J. \& Sussman, M. (1972). Regulation of four functionally related enzymes during shifts in the developmental program of Dictyostelium discoideum. Journal of Molecular Biology 63, 373-382.

Newell, P. C., Henderson, R. F., Mosses, D. \& RAtner, D. I. (1977a). Sensitivity to Bacillus subtilis: a novel system for selection of heterozygous diploids of Dictyostelium discoideum. Journal of General Microbiology 100, 207-211.

Newell, P. C., Ratner, D. I. \& Wright, M. D. $(1977 b)$. New techniques for cell fusion and linkage analysis of Dictyostelium discoideum. In Development and Differentiation in the Cellular Slime Moulds, pp. 51-61. Edited by P. Cappuccinelli \& J. Ashworth. Amsterdam: Elsevier/North Holland.

Perkins, D. D. (1962). Preservation of Neurospora stock cultures with anhydrous silica gel. Canadian Journal of Microbiology 8, 591-594.

Poff, K. L. \& Skokut, M. (1977). Thermotaxis by pseudoplasmodia of Dictyostelium discoideum. Proceedings of the National Academy of Sciences of the United States of America 71, 2007-2010.

Poff, K. L.. Loomis, W. F. \& Burler, W. L. (1974). Isolation and purification of the photoreceptor pigment associated with phototaxis in Dictyostelium discoideum. Journal of Biological Chemistry 249. 2164-2167.

RAPER, K. B. (1940). Pseudoplasmodium formation and organisation in Dictyostelium discoideum. Journal of the Elisha Mitchell Scientific Society 56, 241-282.

Ratner, D. I. \& Newell, P. C. (1978). Linkage analysis in Dictyostelium discoideum using multiply marked tester strains: establishment of linkage group VII and the reassessment of earlier linkage data. Journal of General Microbiology 109, 225-236.

Ross, F. M. \& Newell, P. C. (1979). Genetics of aggregation pattern mutations in the cellular slime mould Dictyostelium discoideum. Journal of General Microbiology 115, 289-300.

Ross, F. M. \& Newell, P. C. (1981). Streamers: chemotactic mutants of Dictyostelium discoideum 
with altered cyclic GMP metabolism. Journal of General Microbiology 127, 339-350.

Schindler, J. \& Sussman, M. (1977). Ammonia determines choice of morphogenetic pathways in Dictyostelium discoideum. Journal of Molecular Biology 116, 161-169.

Schindler, J. \& Sussman, M. (1979). Inhibition by ammonia of intracellular cAMP accumulation in Dictyostelium discoideum. Its significance for the regulation of morphogenesis. Developmental Genetics 1, 13-20.

Slifkin, M. K. \& Bonner, J. T. (1952). The effects of salts and organic solutes on the migration of the slime mold Dictyostelium discoideum. Biological Bulletin 102, 273-277.

Sussman, M., Schindler, J. \& Kim, H. (1978). 'Sluggers', a new class of morphogenetic mutants of Dictyostelium discoideum. Experimental Cell Research 116, 217-227.

Wallace, J. S. \& Newell, P. C. (1982). Genetic analysis by mitotic recombination in Dictyostelium discoideum of growth and developmental loci on linkage group VII. Journal of General Microbiology 128, 953-964.

Welker, D. L. \& Deering, R. A. (1976). Genetic analysis of radiation-sensitive mutations in the slime mould Dictyostelium discoideum. Journal of General Microbiology 97, 1-10.

Welker, D. L. \& Deering, R. A. (1978). Genetics of radiation sensitivity in the slime mould Dictyostelium discoideum. Journal of General Microbiology 109, 11-23.
Welker, D. L. \& Williams, K. L. (1980a). Bacillus subtilis sensitivity loci in Dictyostelium discoideum. FEMS Microbiology Letters 9, 179-183.

Welker, D. L. \& Williams, K. L. $(1980 b)$. The assignment of four new loci, including the coumarin sensitivity locus couA, to linkage group VII of Dictyostelium discoideum. Journal of General Microbiology 120, 149-159.

Welker, D. L. \& Williams, K. L. (1980c). Mitotic arrest and chromosome doubling using thiabendazole, cambendazole, nocodazole and benlate in the slime mould Dictyostelium discoideum. Journal of General Microbiology 116, 397-407.

Williams, K. L. \& Barrand, P. (1978). Parasexual genetics in the cellular slime mold Dictyostelium discoideum: haploidization of diploid strains using benlate. FEMS Microbiology Letters 4, 155-159.

Williams, K. L. \& Newell, P. C. (1976). A genetic study of aggregation in the cellular slime mold Dictyostelium discoideum using complementation analysis. Genetics 82, 287-307.

Williams, K. L., Kessin, R. H. \& Newell, P. C. (1974). Parasexual genetics in Dictyostelium discoideum: mitotic analysis of acriflavin resistance and growth in axenic medium. Journal of General Microbiology 84, 59-69.

Yanagisawa, K., Loomis, W. F. \& Sussman, M. (1967). Developmental regulation of the enzyme UDP-galactose polysaccharide transferase. Experimental Cell Research 46, 328-334. 